\title{
Verification of a timed multitask system with UPPAAL *
}

\author{
Houda BEL MOKADEM \\ LSV - CNRS UMR 8643 \& ENS de Cachan, \\ 61 av. du Prés. Wilson, F-94235 Cachan Cedex, France \\ mokadem@lsv.ens-cachan.fr \\ Béatrice BÉRARD \\ LAMSADE - CNRS UMR 7024 \& Université Paris-Dauphine, \\ Place du Maréchal de Lattre de Tassigny, F-75775 Paris Cedex 16 \\ berard@lamsade.dauphine.fr \\ Vincent GourcufF, Jean-Marc Roussel and Olivier De SMET \\ LURPA - EA 1385 - ENS de Cachan, \\ 61 av. du Prés. Wilson, F-94235 Cachan Cedex, France \\ gourcuff, roussel, de_smet@lurpa.ens-cachan.fr
}

\begin{abstract}
Since it is an important issue for users and system designers, verification of PLC programs has already been studied in various contexts, mostly for untimed programs. More recently, timed features were introduced and modeled with timed automata. In this case study, we consider a part of the so-called MSS (Mecatronic Standard System) platform from Bosh Group, a framework where time aspects are combined with multitask programming. Our model for station 2 of the MSS platform is a network of timed automata, including automata for the operative part and for the control program, written in Ladder Diagram. This model is constrained with atomicity hypotheses concerning program execution and model checking of a reaction time property is performed with the tool UPPAAL.
\end{abstract}

Keywords: Programmable Logic Controllers, Timed Automata, Model Checking.

\section{Introduction}

Verification of safety properties for PLC (Programmable Logic Controller) programs is important when these programs are to control critical applications for reactive systems. This explains the increasing interest in the past few years for the application of formal methods to the analysis of such programs. In this area, work was mostly devoted to the untimed framework [15], [4, 8], even when function blocks for timers were included [16].

${ }^{*}$ This work was supported by the Pluri Formation Project VSMT of ENS Cachan.
Introducing the study of quantitative properties related to time makes this verification step harder, because additional components must be added in the model, for instance clocks, which increase the size of the model. However, the model of timed automata, introduced in 1990 by Alur and Dill [2, 3], has proved very successful. Some decidability results were obtained for this model as well as for some extensions and they were implemented in efficient tools called timed model-checkers, like HYTECH [9], KRONOS [6] or UPPAAL [11], which have been applied to industrial case studies. Timed automata have recently been used for the modeling of timed features in PLC programming $[13,12,7]$.

In this work, we are interested in the combination of time aspects with multitask PLC programming. Our case study concerns a part (called station 2) of the MSS (Mecatronic Standard System) platform from Bosch Group, in which multitask programming can be used to reduce the reaction time of the control program to an external signal. The program is written in Ladder Diagram, one of the languages most commonly used in this area, which is part of the IEC-61131-3 standard [10]. We give semantics for a subclass of Ladder Diagram programs including timer function blocks, in terms of timed automata, and we also provide a timed automata based model for the operative part of the system. These timed automata are described in UPPAAL syntax. While a similar approach was introduced in [12], we propose here additional restrictions which allow us to reduce significantly the size of the complete model, obtained from its components by a synchronized product: these restrictions consist in atomicity hypotheses, compacting sequences of actions from the control program into a single one, and lead to reasonnable verification times for the response property to be checked. 
We also give a simpler model for timers, using particular features of UPPAAL.

Section 2 of the paper explains the context of this study: the problem of reaction times in PLC programs, and includes a description of timed automata and a short presentation of UPPAAL. In Section 3, we give more details on Bosch MSS platform and in Section 4, we give the semantics of the control program. Section 5 presents the timed automata which form the components of the network, while Section 6 gives the results of the verification step.

\section{Programmable Logic Controllers and Timed Automata}

\subsection{Programmable Logic Controllers with multi-task programming}

Programmable Logic Controllers (PLCs) execute programs for the control of an operative part, to which they are connected via an input/output system. The control programs can be written in several languages described in the IEC-61131-3 [10] standard. The execution of such a program consists in iterating a cycle with three main steps (figure 1): first, input variables are read and their values are stored in memory. Then a computation step is performed using these values, producing output values which are also stored. The last step is an activation using the output values. The cycle duration $P$ is called the PLC scan.

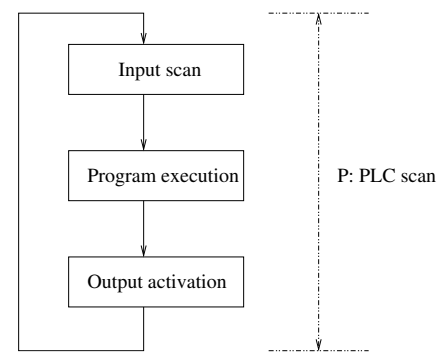

\section{Figure 1. The cyclic execution of a PLC pro- gram}

The programming design may be either monotask or multitask. In the first case, a single program executes sequentially, while in the second case, the main task can be interrupted by additional parts of code, either with a fixed period or triggered by some events. These two execution models result in different reaction times to changes of values. In the monotask case, if the change of value occurs at the input scan, the corresponding output is emitted at the end of the PLC cycle. If the change occurs later, this output may be emitted at as far as the end of the next cycle. This results in a reaction time in the interval $[P, 2 P]$ (figure 2). This reaction time can be reduced with multi-task programming: consider an event-driven task interrupting the main task when some event occurs. In turn, the interrupting task reads its input and computes its new output values. Depending on the configuration and type of the PLC, these values can be emitted either at the end of the event-driven task or at the end of the current main task. In this work, we investigate the second case where output values of the event-driven task are emitted by the main program, which yields a reaction time of at most $P$.

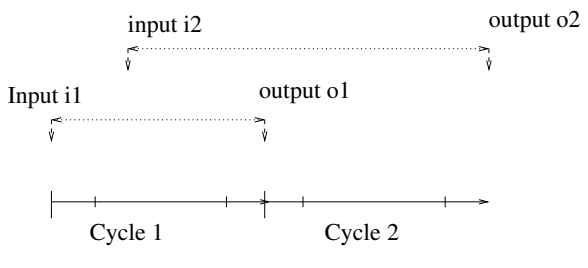

\section{Figure 2. Reaction time with mono-task pro- gramming}

\subsection{Timed automata}

Timed automaton was introduced by Alur and Dill [2], [3]. It consists of finite automaton, which handles a finite set of variables called clocks. The clocks are used for the specification of quantitative time constraints which may be associated with transitions. These variables evolve synchronously with time (slope 1 ).

For a set $X$ of clocks, $\mathcal{P}(X)$ denotes the powerset of $X$ and we define $\mathcal{C}(X)$ as the set of conjunctions of atomic formulas of the form $x \bowtie c$ for a clock $x$, a constant $c$ and $\bowtie$ in $\{<, \leq,=, \geq,>\}$.

A timed automaton is a tuple $\mathcal{A}=\left(\Sigma, X, Q, q_{0}, I, E\right)$, where $\Sigma$ is a finite set of actions, $X$ is the finite set of clocks, $Q$ is a finite set of locations, with $q_{0} \in Q$ the initial location, $I$ is a mapping associating with each location $q$ a clock constraint $I(q) \in \mathcal{C}(X)$, and $E \subseteq Q \times \mathcal{C}(X) \times$ $\Sigma \times \mathcal{P}(X) \times Q$ is the set of transitions.

The clock condition $I(q)$ is called an invariant for location $q$, and contains usually only atomic formulas of the form $x \leq c$ or $x<c$ which must hold as long as time elapses in this location.

A transition of the automaton, written $q \stackrel{g, a, r}{\longrightarrow} q^{\prime} \in E$ is equipped with a label containing three parts (each one is optional): a guard $g$ expressing a condition in $\mathcal{C}(X)$ on clock values, which must be satisfied for the transition to be fired, an action name in $\Sigma$, and a clock reset $r \in \mathcal{P}(X)$.

The semantics of a timed automaton is given in terms of transition systems. A configuration of the system is a pair $(q, v)$, where $q$ is a location of the automaton and $v$ is a valuation of the variables, i.e. a mapping associating a real value with each clock. The initial configuration is $\left(q_{0}, v_{0}\right)$ where all clock values are equal to 0 in $v_{0}$.

The system may change its configuration in two ways.

- Either by a delay move of $d$ time units, written $(q, v) \stackrel{d}{\rightarrow}(q, v+d)$, possible if $v+d$ satisfies the invariant $I(q)$ of location $q$. 
- Or by an action move, written $(q, v) \stackrel{a}{\rightarrow}\left(q^{\prime}, v^{\prime}\right)$, associated with a discrete transition $q \stackrel{g, a, r}{\longrightarrow} q^{\prime}$, if $v$ satisfies the constraint $g$. In this case, the reset operation yields $v^{\prime}(x)=0$ if $x$ belongs to $r$ and $v^{\prime}(x)=v(x)$ otherwise, and $v^{\prime}$ must satisfy the invariant of $q^{\prime}$.

\subsection{The tool Uppaal}

The tool UPPAAL (see [5] for the more recent developments) offers a compact description language, a simulation module and a model-checker. A system is represented by a collection for timed automata, which communicate through binary synchronization: a channel $c$ can be defined for two automata. Sending a message is denoted by the discrete action $c$ ! while receiving the message is denoted by $c$ ?. An UPPAAL automaton also handles integer variables. A guard is a conjunction of atomic clock conditions and similar conditions on integer variables. Moreover, a clock reset may be augmented by an update of the integer variables.

A (global) configuration is of the form $(\ell, v)$ where $\ell$ is a location vector (indicating the current state in each component of the timed automata network) and $v$ is a valuation of both clocks and discrete variables. An execution in the network starts in initial locations of the different components with all the clocks and variables set to zero. The semantics of this model is expressed by moves between the configurations. Three types of moves can occur in the system: delay moves, internal moves and synchronized moves. Delay moves and internal moves have already been described above for a single automaton, so we simply describe now the global evolution.

Delaying. Given a current location vector, time elapses for all automata synchronously, as long as no invariant is violated. All clock values increase by the amount of time elapsed. No change occur for the locations or the integer variables.

Performing an internal action. An internal action is an action which corresponds to neither $c$ ! (sending a message), nor $c$ ? (receiving a message). If such an action is enabled (the variable values satisfy the guard condition), the component can perform this action alone, while the others do nothing. Only the location of this component is changed, as well as its variables, according to the transition.

Synchronizing. If, in the network, some complementary actions $c$ ! and $c$ ? are enabled in two components (in particular, guards must be satisfied by the current valuation), then these components must synchronize. The location vector is changed for both components and the clock and variable values are changed according to the clock reset and updates of variables for the two transitions.

Finally, we introduce two additional features of UPPAAL which will be very useful in our modeling.

- A committed location (decorated by the special label C) corresponds to a location in which no delay move is possible. Only a discrete transition can be used to leave such a location. Note that this mechanism reduces the non-determinism in the parallel composition of the different components.

- A broadcast channel is a channel where more than two automata may communicate: emission of a message $c$ ! can be synchronized with several receptions $c$ ? in other components. Note that this is a nonblocking synchronization, since the sender is never blocked, although the receiver must synchronize if it can. Guards on clocks are not allowed on the receiving edge.

\section{Description of the MSS (Mecatronic Stan- dard System) platform}

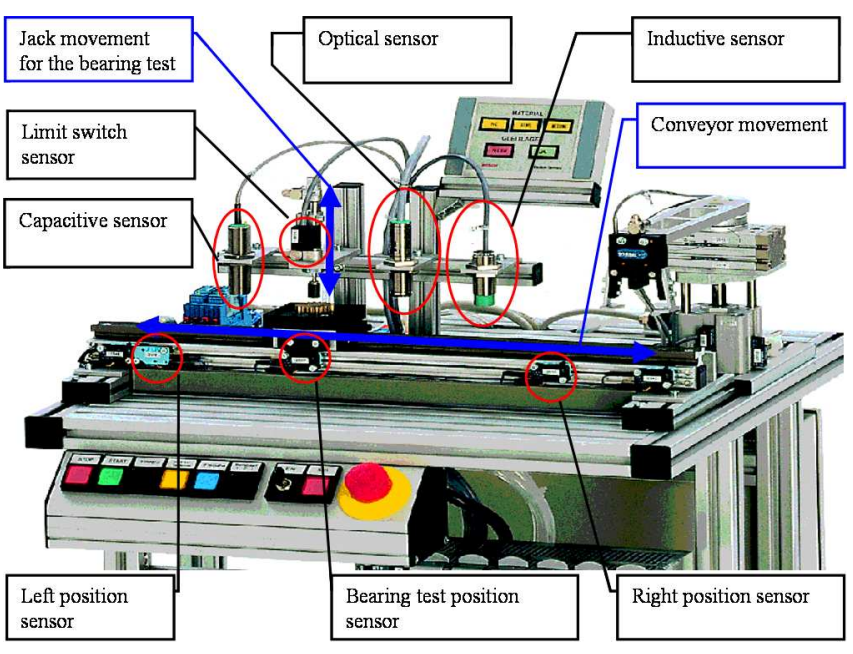

Figure 3. presentation of station 2 of the
MSS platform

Presentation. Platform MSS (from Bosch Group) provides a function for sorting a stock of pinions of different materials and for adding or withdrawing a press-fit bushing to a given pinion [14].

Our study is centered on station 2 (figure 3 ) which is intended to identify the material of the pinion (steel, copper or black PVC) and the presence or absence of a pressfit bushing. The workpieces are transported by a linear conveyor to a scanning position, where the presence or absence of a press-fit bushing is detected. They are then tested by three sensors to determine their material. This is done using inductive, capacitive and optical sensors. The detected information is forwarded to the next stations. A rotary/lift gripper performs the transfer to a follow-on station if applicable.

Issue detected. A problem arises when the conveyor arrives at the bearing test position (POS_TEST sensor). At this time the conveyor moves at high speed $(200 \mathrm{~mm} / \mathrm{s})$ 
and the variation of the reaction time of the control system, above $10 \mathrm{~ms}$, is not negligible. Indeed the conveyor position should have a precision of $1 \mathrm{~mm}$ for the tester (or jack) to be able to penetrate inside the pinion, in case the bearing is absent. So, we can deduce that the the variation of the reaction time of the control system must be less than $5 \mathrm{~ms}$. In the rest of the paper, we study the case of a multitask controller, with an event-driven task, launched on the rising edge of the test position (POS_TEST) sensor, which stops the conveyor if it comes from the loading station.

Properties to check. The multitask control program of this station must satisfy the following properties:

P1 To ensure safety, the conveyor must stop on its way out but not when it comes back from unloading.

P2 The time performance is accurate: the conveyor stops in less than $5 \mathrm{~ms}$ at the press-fit bushing test point.

In this work, we focus on the timed property $\mathbf{P 2}$, to show that the multitask solution reduces the reaction time.

\section{Modeling principles}

In this section, we briefly recall the timed automaton based semantics proposed by Mader and Wupper [12] for a control program. Then we explain the structure of our model for (station 2 of) the MSS platform, with a particular attention to the question of timers.

\subsection{Mader-Wupper model}

Various models have already been proposed for the analysis of PLC programs. Our approach is based on the model introduced by Mader and Wupper [12], which disregards the execution times of elementary instructions.
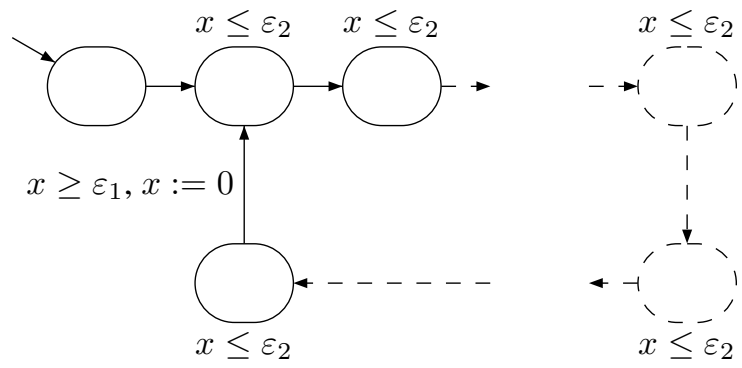

Figure 4. Mader-Wupper model

As depicted in figure 4, the model has a clock $x$ to measure the cycle scan, which is thus reset after each cycle of the program. The invariant $x \leq \varepsilon_{2}$ is associated with each location and represents an upper time bound for each whole scan. The guard $x \geq \varepsilon_{1}$ appears on the last edge of the cycle and represents a lower time bound for the input/output part of the cycle. An edge in the model describes a step of the control program.
Mader-Wupper also models each timer block as a timed automaton that runs in parallel with the control program. Synchronization is performed through operations on the timer variables and on the timer calls, which requires one extra clock and three synchronization channels for each timer.

\subsection{An overview of the model}

Our model is built in a compositional way from a collection of non deterministic processes with finite control structure and real-valued clocks, communicating through channels or shared variables. The two main parts are the environment and the control program, which communicate through shared variables and synchronization messages. The modeling of the operative part (environment) is necessary for the verification of the safety and performance properties stated previously. The details are explained in Section 5.

\subsection{Modeling timers}

Six independent timers (TON function block in IEC61131-3 [10]) are used in station 2 control program. We now explain how our model of a TON function block differs from that of Mader-Wupper [12] and how we used broadcast channels in UPPAAL to avoid deadlocks. Each TON block is modeled by an automaton, described in figure 5, with three states, one clock $x$-Ton and two discrete variables Ton-ine (input) and Ton-Qe (output). Initially idle, the state becomes running when the timer has been switched on and Timeout, when some fixed preset delay (constant Ton_pte) has been reached. At each cycle of the main task, a synchronization message is sent. The automaton is then forced to evolve by taking into account the new values of the variables computed by the previous cycle. A deadlock could occur if the automaton is in a state where it cannot receive the synchronization message of the program. So we chose a single broadcast channel (with emission TON! and reception TON?) for all TON blocks instead of three ordinary channels per TON in Mader-Wupper's model.

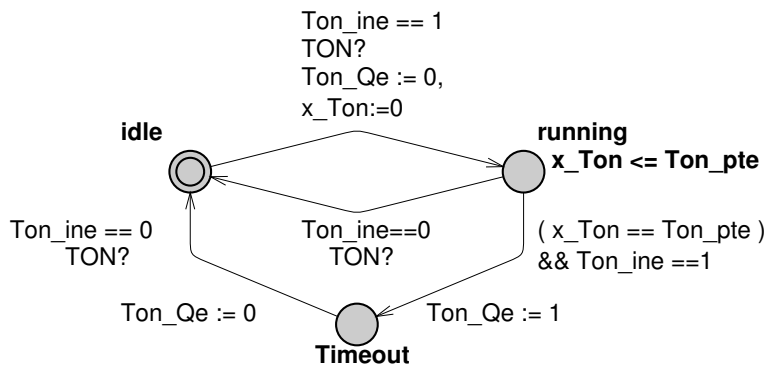

Figure 5. UPPAAL model of a TON block 


\section{Modeling with UPPAAL}

\subsection{Modeling the environment}

Interest. In order to validate not only the PLC program but also its integration in the system it has to control, we also need to model the operative part. This implies a thorough knowledge of the system to control, particularly the behavior of each element and its reaction time. Modeling the environment makes it possible to speed-up the verification time, in particular by reducing the combinatorial aspects related to non deterministic definition of all possible input values, including sometimes non relevant ones. Indeed, when the input values of the PLC program are emitted by a model instead of a non deterministic process, the space of reachable states is reduced. However, these parts of the model are usually limited to the representation of nominal operation modes, which is the case here.

Modeling. Each physical device is represented by a timed automaton. In such an automaton, a given location represents a particular configuration of the device. In the models proposed here, clocks are the only continuous components, while physical continuous moves are discretized (for instance for the conveyor).

The external environment. In station 2, the leftmost position corresponds to the loading of pinions, while the rightmost position is used for unloading. However, the control of loading and unloading operations is not part of this station, which just waits for them to be done. Information about termination of one of these operations is obtained through changes of input values. Upon loading, the conveyor is provided an unspecified pinion. This is modelled by an automaton, presented in figure 6 , which selects in a non deterministic way the nature of the pinion (variable $o b$ ) when the conveyor is at the rightmost position.

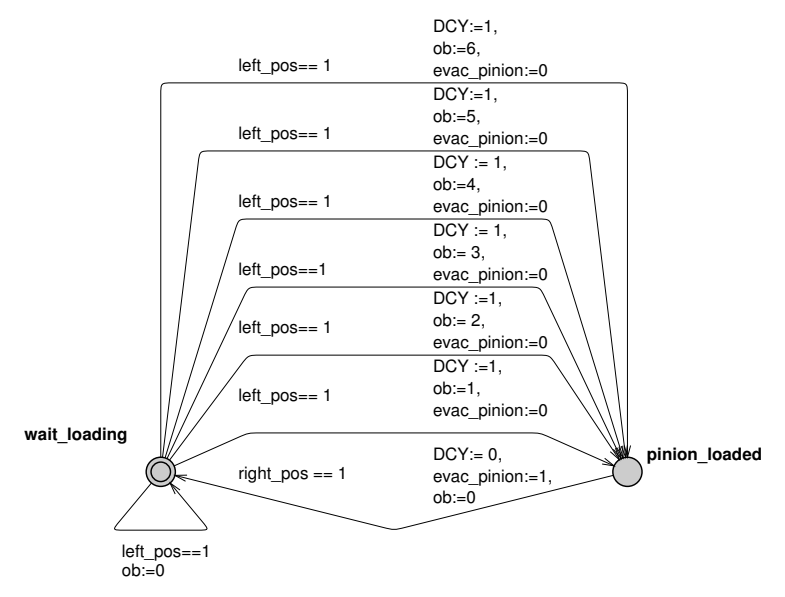

Figure 6. Model of the environment external to station 2

The jack. The jack detects the presence or absence of a press-fit bushing in a workpiece. This test is made by a vertical movement of the jack until a limiting posi-

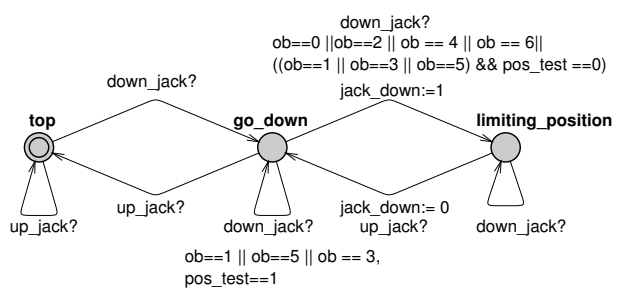

Figure 7. Timed automaton for the jack.

tion. The jack must go down until the limiting position is reached, in a given time, to conclude to the absence of the press-fit bushing. The model of this sensor (figure 7) depends on the characteristics of the workpieces which are represented by the values of the variable $o b$. The automaton starts from the state top. He moves to state go down when he receives a message down-jack? from the PLC program. From this point on, there are two cases: if there is a press-fit bushing in the workpiece (represented in the model by the guard $o b==1\|o b==3\| o b==5$ ) then the automaton waits in the state go down, else the automaton moves to state limiting position.

The sensors. The optical, capacitive and inductive sensors are modeled by automata synchronized with the automaton of the conveyor. The conveyor sends the activation messages (for example optics? in figure 8) when it is under the corresponding sensor. According to the nature of the material, the sensor modifies the value of the corresponding variable (optical) which is then used by the PLC program.

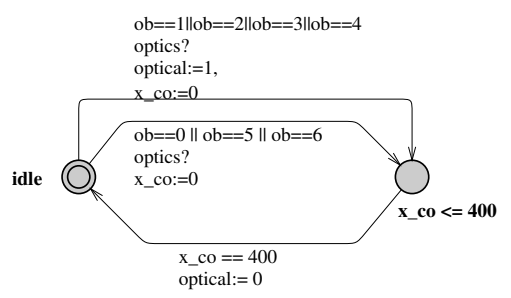

Figure 8 . Timed automaton for the optical
sensor.

The linear conveyor. The conveyor is the main element of the operative part: several triggerings of sensor depend on its position. The conveyor is also the most delicate to model because of its continuous behavior along the belt, while our model can only provide a discrete abstraction of this behavior, leaving out the details which do not influence the properties to be checked. In order to obtain reasonable performances in terms of memory and automatic verification time, we model only the almost stable positions, i.e. the positions where the conveyor can stop, or trigger a sensor. These positions correspond to the six states: inductivesensor, capacitivesensor, optical- 


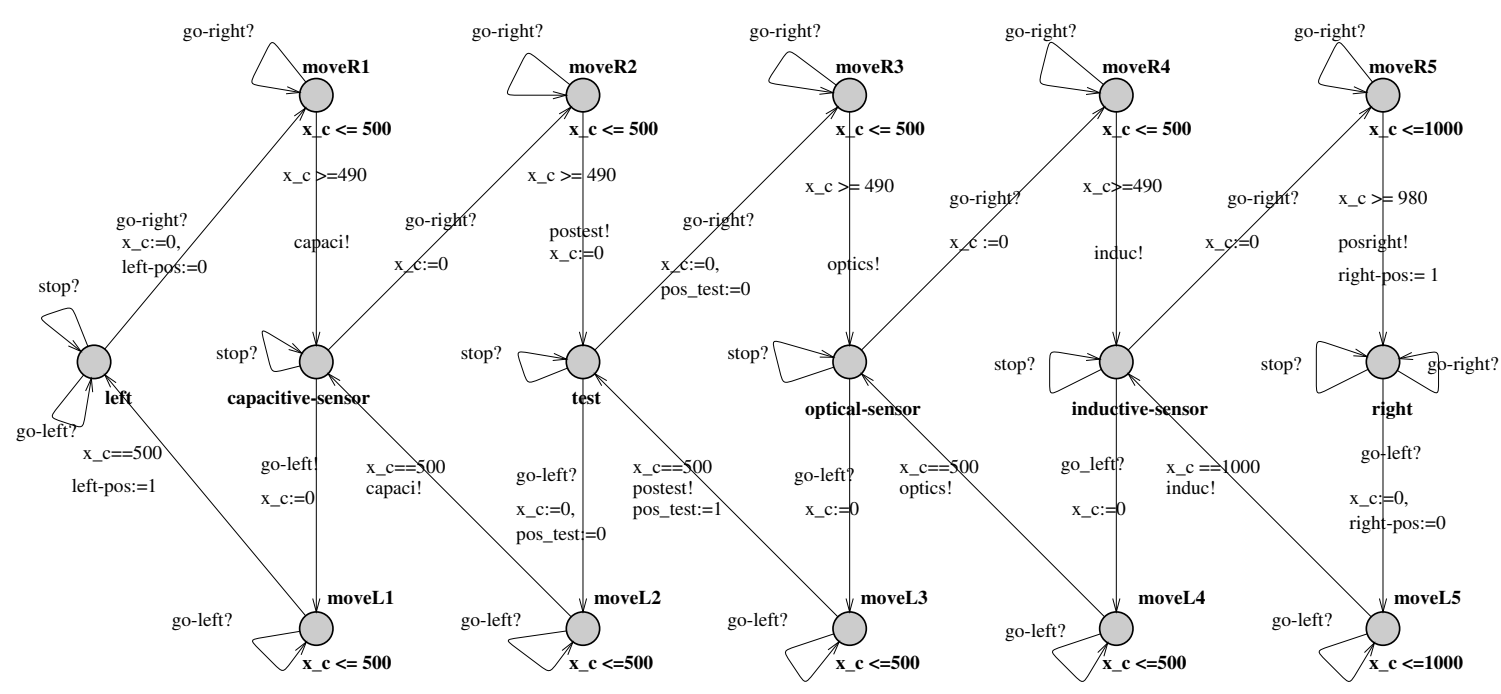

Figure 9. Timed automaton for the conveyor.

sensor, test, left, right. Between two given positions, we model the behavior of the conveyor by only one state with an invariant which represents the time needed by the conveyor to cross the distance between these two positions. For example, the conveyor goes from the left position to the capacitive-sensor position in 490 to $500 \mathrm{~ms}$. There is another abstraction imposed by the fact that no stopwatch exists in UPPAAL: between two almost stable positions, the conveyor cannot change direction. The conveyor sends messages of synchronization to the various sensors (like optics!) and the event-driven task (postest!) at the time of its arrival to the test position. It also modifies the input variables of the control program. The corresponding automaton is represented in figure 9.

\subsection{The control program}

The main program. The functional specification of the global system is designed in GRAFCET (or SFC) language, and further implemented in Ladder language. As explained above, the execution of a PLC program is a cycle with three phases: input reading, computation of new values and output writing. This periodic operation is modeled in UPPAAL by an automaton structured as a loop, and including a clock to measure the cycle time (equal to 10 u.t. here). The complete cycle of the automaton for the ladder program thus consists of a loop with four steps:

1. input reading and computation of new values for the evolution conditions of the GRAFCET,

2. computation of other new values for GRAFCET variables: step activation and output computation,

3. output writing, performed by a sequence of messages for synchronization with the operative part,

4. reset of the clock modeling the cycle time.
The atomicity hypothesis is the following: time can elapse only in the three states between these steps, to represent the duration of their execution.

The event-driven program. Since it is run upon activation of the bushing-test position, the event-driven task is strongly dependent on the environment. This aspect is modeled by the emission of a message from the environment, received by the automaton of the event-driven task (figure 11).

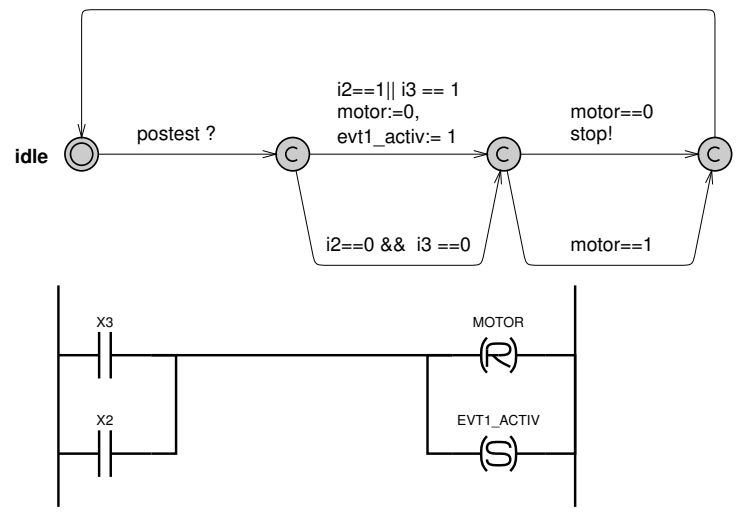

Figure 11. UPPAAL model and Ladder Diagram for the event-driven task

When the message postest! is emitted, the automaton executes the algebraic equations which represent the Ladder program and sends the output message stop! if the condition holds. Note that the execution time of the evendriven task is null due to the committed location used to model the priority of the event-driven task. Various programming designs are considered in order to determine the conditions under which the requirements are satisfied:

- the event-driven task emits his own output, 


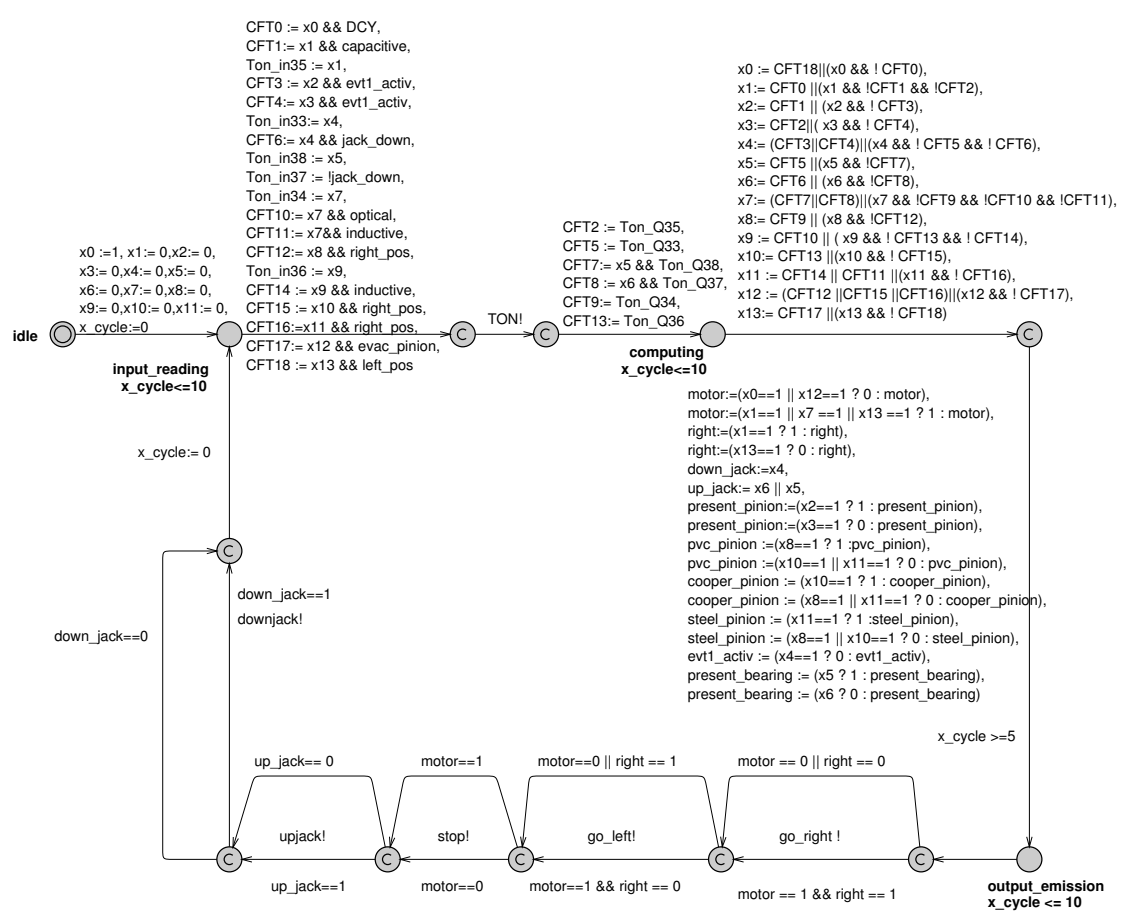

Figure 10. UPPAAL model of main program

- the event-driven task only modifies the internal memory of the output,

- the event-driven task is not activated.

\section{Verification with UPPAAL}

The observer automaton. In order to verify the timed property P2, we need an additional automaton (see below), which plays the role of an external observer with respect to the model previously described.

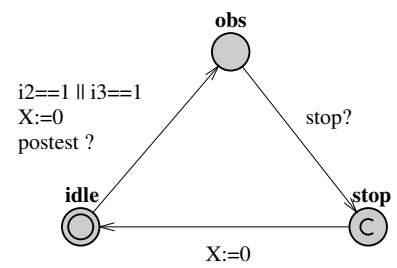

This automaton contains a state stop, reached when the conveyor stops in testing position. It also contains a clock $X$ to measure the reaction time. The observer automaton starts from state idle with $X$ set to 0 . When the message postest? is received from the conveyor, the automaton moves to state $o b s$ and resets the clock $X$. From this point on, the clock value again increases with time. When the message stop? is received from the main program, the automaton switches to state stop. Thus, the value of $X$ in this last state corresponds to the time elapsed between the triggering of the event-driven task and the physical stop of the conveyor. To check the timed property $\mathbf{P 2}$, we express its negation ( $\mathrm{C} 1$ in the table below): the observer automaton will eventually reach the state stop with the value of the clock $X$ greater than 5 time units. This property is written as

$$
\mathrm{E}<>\text { (obs.stop and } X>5)
$$

in UPPAAL syntax, which is a fragment of the logic TCTL [1]. In this formula, the combination $\mathrm{E}<>$ means "for some path in the future" and obs.stop denotes location stop of the observer automaton.

Experiments. First note that the global model has about $30.10^{6}$ configurations, which are explored in an on the fly computation of the set of reachable states. The table below gives the time and memory used for verification (on a linux machine with a pentium 4 at $2.4 \mathrm{GHz}$ with $3 \mathrm{Go}$ RAM). The results provide a comparison of the reaction times between monotask and multitask programming. Indeed, on one hand, properties C5, C6 and C7 show that the conveyor stops between 10 and 20 time units after it reaches the test position. This is far from being a surprise because these values correspond respectively to one and two PLC cycle times. On the other hand, property C3 shows that the conveyor stops in less than one PLC cycle time. So, multitask programming reduces the reaction time. However, property $\mathrm{C} 1$ proves that it is not sufficient to satisfy the requirement $\mathbf{P 2}$.

Note that, after 29 hours of computation, we stopped the verification process in the case of Mader-Wupper model. 


\begin{tabular}{|l|c|c|c|}
\hline property & result & time & memory \\
\hline with the event driven task & & & \\
$\mathrm{C} 1: \mathrm{E}<>$ obs.stop and $X>5$ & yes & $15 \mathrm{~s}$ & $30 \mathrm{Mo}$ \\
$\mathrm{C} 2: \mathrm{E}<>$ obs.stop and $X \leq 5$ & yes & $15 \mathrm{~s}$ & $30 \mathrm{Mo}$ \\
$\mathrm{C} 3: \mathrm{E}<>$ obs.stop and $X>10$ & no & $22 \mathrm{~s}$ & $61 \mathrm{Mo}$ \\
& & & \\
\hline without the event driven task & & & \\
& & & \\
$\mathrm{C} 5: \mathrm{E}<>$ obs.stop and $X \geq 10$ & yes & $16 \mathrm{~s}$ & $30 \mathrm{Mo}$ \\
$\mathrm{C} 6: \mathrm{E}<>$ obs.stop and $X>20$ & no & $22 \mathrm{~s}$ & $70 \mathrm{Mo}$ \\
$\mathrm{C} 7: \mathrm{E}<>$ obs.stop and $X<10$ & no & $22 \mathrm{~s}$ & $69 \mathrm{Mo}$ \\
& & & \\
\hline with Mader-Wupper model & & & \\
C8:E $<>$ obs.stop and $X>5$ & - & - & - \\
\hline
\end{tabular}

These performances are due to two main reasons: the atomicity hypothesis for executions between some states of the main program and the enhanced model of the TON block.

- The atomicity hypothesis: we assume that each one of the four steps of the main program (section 5.2) executes instantaneously. Recall that time can elapse only in three states.

- The enhanced model of the TON block: we use one broadcast channel to synchronize all the TON blocks and the main program instead of three ordinary channels for each TON block as in Mader-Wupper model.

\section{Conclusion}

In this work, we give formal semantics to (partial) Ladder diagrams and TON blocks, with timed automata. We also describe the operative part of station 2 of MSS platform with timed automata. On this network of timed automata represented in UPPAAL syntax, we formally prove by model-checking that multitask programming reduces the reaction time of the conveyor, upon emission of an output order to stop. While this does not really come as a surprise, we obtain reasonable verification times (less than 30 s) on a global model with about $30.10^{6}$ states, by adding an atomicity hypothesis to Mader-Wupper model and modifying the automata for timer blocks. In comparison, model-checking the same formula with the original model had to be stopped after several hours.

\section{References}

[1] R. Alur, C. Courcoubetis, and D. L. Dill. Modelchecking in dense real-time. Information and Computation, 104(1):2-34, 1993.

[2] R. Alur and D. L. Dill. Automata for modeling real-time systems. In Proc. 17th Int. Coll. Automata, Languages, and Programming (ICALP'90), Warwick University, England, July 1990, volume 443 of Lecture Notes in Computer Science, pages 322-335. Springer, 1990.
[3] R. Alur and D. L. Dill. A Theory of Timed Automata. Theoretical Computer Science (TCS), 126(2):183-235, 1994.

[4] G. Canet, S. Couffin, J.-J. Lesage, A. Petit, and Ph. Schnoebelen. Towards the automatic verification of PLC programs written in Instruction List. In Proc. IEEE Int. Conf. Systems, Man and Cybernetics (SMC'2000), Nashville, TN, USA, Oct. 2000, pages 2449-2454, 2000.

[5] A. David, G. Behrmann, K. G. Larsen, and W. Yi. A Tool Architecture for the Next Generation of UPPAAL. Technical Report 2003-011, Department of Information Technology, Uppsala University, Feb. 2003. 20 pages.

[6] C. Daws, A. Olivero, S. Tripakis, and S. Yovine. The tool KRONOS. In Proc. Workshop Hybrid Systems III: Verification and Control, New Brunswick, NJ, USA, Oct. 1995, volume 1066 of Lecture Notes in Computer Science, pages 208-219. Springer, 1996.

[7] H. Dierks. PLC-Automata: A New Class of Implementable Real-Time Automata. Theoretical Comput. Sci., 253(1):61-93, 2000.

[8] G. Frey and L. Litz. Formal methods in PLCprogramming. In Proc. IEEE Int. Conf. Systems, Man and Cybernetics (SMC'2000), Nashville, TN, USA, Oct. 2000, pages 2431-2436, 2000.

[9] T. A. Henzinger, P.-H. Ho, and H. Wong-Toi. A user guide to HyTech. In Proc. 1st Int. Workshop Tools and Algorithms for the Construction and Analysis of Systems (TACAS'95), Aarhus, DK, May 1995, volume 1019 of Lecture Notes in Computer Science, pages 41-71. Springer, 1995.

[10] IEC (International Electrotechnical Commission). IEC Standard 61131-3 : Programmable controllers - Part 3, 1993.

[11] K. G. Larsen, P. Pettersson, and W. Yi. UPPAAL in a nutshell. Journal of Software Tools for Technology Transfer, 1(1-2):134-152, 1997.

[12] A. Mader and H. Wupper. Timed automaton models for simple programmable logic controllers. In Proc. 11th Euromicro Conference on Real-Time Systems (ECRTS'99), York, UK, June 1999, pages 114-122. IEEE Comp. Soc. Press, 1999.

[13] E. Olderog. Correct real-time software for programmable logic controllers. In Correct System Design. Recent Insights and Advances, volume 1710 of Lecture Notes in Computer Science, pages 342-362. Springer, 1999.

[14] Rexroth Bosch Group. Mechatronik standard system. http: / / www.boschrexroth. com/country_ units/europe/germany/sub_websites/ brs_germany/de/didactic/lehrsysteme/ mechatronik/mechatronik_standard_ system.mss/index.jsp.

[15] O. Rossi, O. de Smet, S. Lamprire-Couffin, J.-J. Lesage, H. Papini, and H. Guennec. Formal verification: a tool to improve the safety of control systems. In 4th Symposium on Fault Detection, Supervision and Safety for Technical Processes (IFAC Safeprocess 2000), Budapest, Hungary, pages 885-890, 2000.

[16] O. Rossi and Ph. Schnoebelen. Formal modeling of timed function blocks for the automatic verification of Ladder Diagram programs. In Proc. 4th Int. Conf. Automation of Mixed Processes: Hybrid Dynamic Systems (ADPM'2000), Dortmund, Germany, Sept. 2000, pages 177-182. Shaker Verlag, Aachen, Germany, 2000. 